\title{
A METHOD OF UPPER AND LOWER SOLUTIONS FOR FUNCTIONAL DIFFERENTIAL INCLUSIONS
}

\author{
MOUFFAK BENCHOHRA \\ University of Sidi Bel Abbés \\ Department of Mathematics \\ BP 89, 22000 Sidi Bel Abbés, Algeria \\ E-mail: benchohra@yahoo.com \\ SOTIRIS K. NTOUYAS \\ University of Ioannina \\ Department of Mathematics \\ 45110 Ioannina, Greece \\ E-mail: sntouyas@cc.uoi.gr
}

(Received February, 2001; Revised January, 2002)

In this paper, a fixed point theorem for condensing maps combined with upper and lower solutions are used to investigate the existence of solutions for first order functional differential inclusions.

Key words: Initial Value Problem, Convex Multivalued Map, Functional Differential Inclusions, Condensing Map, Fixed Point, Truncation Map, Upper and Lower Solutions.

AMS subject classifications: 34A60, 34K10.

\section{Introduction}

This paper is concerned with the existence of solutions for the initial multivalued problem:

$$
\begin{gathered}
y^{\prime} \in F\left(t, y_{t}\right) \text {, for a.e. } t \in J=[0, T] \\
y_{0}=\phi,
\end{gathered}
$$

where $F: J \times C\left(J_{0}, \mathbb{R}\right) \rightarrow 2^{\mathbb{R}}\left(J_{0}=[-r, 0]\right)$ is a nonempty compact and convex valued multivalued map, $\phi \in C\left(J_{0}, \mathbb{R}\right)$.

For any continuous function $y$ defined on the interval $J_{1}=[-r, T]$ and any $t \in J$, we denote by $y_{t}$ the element of $C\left(J_{0}, \mathbb{R}\right)$ defined by

$$
y_{t}(\theta)=y(t+\theta) \quad \theta \in J_{0} .
$$

Here $y_{t}(\cdot)$ represents the history of the state from time $t-r$, up to the present time $t$.

The method of upper and lower solutions has been successfully applied to study the existence of multiple solutions for initial and boundary value problems of first order functional differential equations. 
This method has been used only in the context of single-valued, functional differential equations. We refer to the papers of Haddock and Nkashama [6], Hristova and Bainov [10], Liz and Nieto [13], and Nieto, Jiang and Jurang [15]. For other results on functional differential equations using other methods, we refer to the books of Erbe, Qingai and Zhang [5], Hale [7], Henderson [9], and the survey paper of Ntouyas [16]. Notice that very recently this method has been used for initial and boundary value problems for differential inclusions in the papers of Benchohra and Boucherif [2], Benchohra and Ntouyas [3], and Halidias and Papageorgiou [8].

In this paper we establish an existence result for the problem (1)-(2). Our approach is based on the existence of upper and lower solutions and a fixed point theorem for condensing maps developed by Martelli [14].

\section{Preliminaries}

We will briefly recall some basic definitions and facts from multivalued analysis that we will use in the sequel.

$A C(J, \mathbb{R})$ is the space of all absolutely continuous functions $y: J \rightarrow \mathbb{R}$.

$C\left(J_{1}, \mathbb{R}\right)$ is the Banach space of all continuous functions $y: J_{1} \rightarrow \mathbb{R}$ normed by

$$
\|y\|_{\infty}=\sup \left\{|y(t)|: t \in J_{1}\right\} \text { for each } y \in C\left(J_{1}, \mathbb{R}\right) .
$$

Set $\quad E:=C\left(J_{1}, \mathbb{R}\right) \cap A C(J, \mathbb{R}) . \quad$ For $\quad y, \bar{y} \in E_{0}:=\left\{y \in E: y(t)=\phi(t), \forall t \in J_{0}\right\}$ condition

$$
y \leq \bar{y} \text { if and only if } y(t) \leq \bar{y}(t) \text { for all } t \in J_{1}
$$

defines a partial ordering in $E_{0}$. If $\alpha, \beta \in E_{0}$ and $\alpha \leq \beta$, we denote

$$
[\alpha, \beta]=\left\{y \in E_{0}: \alpha \leq y \leq \beta\right\} .
$$

Let $(X,\|\cdot\|)$ be a normed space. A multivalued map $G: X \rightarrow 2^{X}$ is convex (closed) valued if $G(x)$ is convex (closed) for all $x \in X$. $G$ is bounded on bounded sets if $G(B)=\bigcup_{x \in B} G(x)$ is bounded in $X$ for all bounded subsets $B$ of $X$ (i.e., $\left.\sup _{x \in B}\{\sup \{\|y\|: y \in G(x)\}\}<\infty\right)$. $G$ is called upper semi-continuous (u.s.c.) on $X$ if for each $x_{0} \in X$ the set $G\left(x_{0}\right)$ is a nonempty, closed subset of $X$, and, if for each open set $N$ of $X$ containing $G\left(x_{0}\right)$, there exists an open neighborhood $M$ of $x_{0}$ such that $G(M) \subseteq N$.

$G$ is said to be completely continuous if $G(B)$ is relatively compact for every bounded subset $B \subset X$.

If the multivalued map $G$ is completely continuous with nonempty compact values, then $G$ is u.s.c. if and only if $G$ has a closed graph (i.e., $x_{n} \rightarrow x_{*}, y_{n} \rightarrow y_{*}, y_{n} \in G\left(x_{n}\right)$ imply $\left.y_{*} \in G\left(x_{*}\right)\right)$.

$G$ has a fixed point if there is $x \in X$ such that $x \in G(x)$. $X$.

In the following, $C C(X)$ denotes the set of all nonempty compact and convex subsets of

An upper semi-continuous map $G: X \rightarrow 2^{X}$ is said to be condensing [14] if for any bounded subset $N \subseteq X$ with $\mu(N) \neq 0$, we have $\mu(G(N))<\mu(N)$, where $\mu$ denotes the Kuratowski measure of noncompactness [1]. We remark that a compact map is the easiest example of a condensing map. For more details on multivalued maps, see the books of Deimling [4] and $\mathrm{Hu}$ and Papageorgiou [11].

The multivalued map $F: J \rightarrow C C(\mathbb{R})$ is said to be measurable if, for every $y \in \mathbb{R}$, the function $t \mapsto d(y, F(t))=\inf \{\|y-z\|: z \in F(t)\}$ is measurable. 
Definition 2.1: A multivalued map $F: J \times C\left(J_{0}, \mathbb{R}\right) \rightarrow 2^{\mathbb{R}}$ is said to be an $L^{1}$-Carathéodory if

(i) $\quad t \mapsto F(t, u)$ is measurable for each $u \in C\left(J_{0}, \mathbb{R}\right)$;

(ii) $\quad u \mapsto F(t, u)$ is upper semicontinuous for almost all $t \in J$;

(iii) For each $k>0$, there exists $\varphi_{k} \in L^{1}\left(J, \mathbb{R}_{+}\right)$such that

$$
\begin{gathered}
\|F(t, u)\|=\sup [|v|: v \in F(t, u)] \leq \varphi_{k}(t) \text { for all } \\
\|u\| \leq k \text { and for almost all } t \in J .
\end{gathered}
$$

Let us start $b$ defining what we mean by a solution of the problem (1)-(2).

Definition 2.2: A function $y \in E$ is said to be a solution of (1)-(2) if there exists a function $v \in L^{1}(J, \mathbb{R})$ such that $v(t) \in F\left(t, y_{t}\right)$ a.e. on $J, y^{\prime}(t)=v(t)$ a.e. on $J$ and $y_{0}=\phi$.

The following concept of lower and upper solutions for (1)-(2) was introduced by Halidias and Papageorgiou in [8] for second order multivalued boundary value problems. It will be the basic tool in the approach that follows.

Definition 2.3: A function $\alpha \in E_{0}$ is said to be a lower solution of (1)-(2) if there exists $v_{1} \in L^{1}(J, \mathbb{R})$ such that $v_{1}(t) \in F\left(t, \alpha_{t}\right)$ a.e. on $J, \alpha^{\prime}(t) \leq v_{1}(t)$ a.e. on $J$. Similarly, a function $\beta \in E_{0}$ is said to be an upper solution of (1)-(2) if there exists $v_{2} \in L^{1}(J, \mathbb{R})$ such that $v_{2}(t) \in F\left(t, \beta_{t}\right)$ a.e. on $J, \beta^{\prime}(t) \geq v_{2}(t)$ a.e. on $J$.

For the multivalued map $F$ and for each $y \in C\left(J_{1}, \mathbb{R}\right)$ we define $S_{F, y}^{1}$ by

$$
S_{F, y}^{1}=\left\{v \in L^{1}(J, \mathbb{R}): v(t) \in F\left(t, y_{t}\right) \text { for a.e. } t \in J\right\} \text {. }
$$

Our main result is based on the following:

Lemma 2.4: [12] Let $X$ be a Banach space and $J$ a real compact interval. Let $F: J \times X \rightarrow C C(X)$ be an $L^{1}$-Carathéodory multivalued map with $S_{F}^{1} \neq \emptyset$ and let $\Gamma$ be a linear continuous mapping from $L^{1}(J, X)$ to $C(J, X)$, then the operator

$$
\Gamma \circ S_{F}^{1}: C(J, X) \rightarrow C C(C(J, X)), y \mapsto\left(\Gamma \circ S_{F}^{1}\right)(y):=\Gamma\left(S_{F, y}^{1}\right)
$$

is a closed graph operator in $C(J, X) \times C(J, X)$.

Lemma 2.5: [14] Let $G: X \rightarrow C C(X)$ be an u.s.c. and condensing map. If the set

$$
M:=\{v \in X: \lambda v \in G(v) \text { for some } \lambda>1\}
$$

is bounded, then $G$ has a fixed point.

\section{Main Result}

We are now in a position to state and prove our result for the problem (1)-(2).

Theorem 3.1: Suppose $F: J \times C\left(J_{0}, \mathbb{R}\right) \rightarrow C C(\mathbb{R})$ is an $L^{1}$-Carathéordory multivalued map which satisfies the condition

$(H)$ there exist $\alpha$ and $\beta$ in $E_{0}$ lower and upper solutions, respectively, for the problem (1)-(2) such that $\alpha \leq \beta$.

Then the problem (1)-(2) has at least one solution $y \in E$ such that

$$
\alpha(t) \leq y(t) \leq \beta(t) \text { for all } t \in J_{1} .
$$

Proof: Set 


$$
C_{0}\left(J_{1}, \mathbb{R}\right):=\left\{y \in C\left(J_{1}, \mathbb{R}\right): y(t)=\phi(t), t \in J_{0}\right\} .
$$

Transform the problem into a fixed point problem. Consider the following modified problem

$$
\begin{gathered}
y^{\prime}(t) \in F\left(t,(\tau y)_{t}\right), \text { a.e. } t \in J, \\
y_{0}=\phi,
\end{gathered}
$$

where $\tau: C_{0}\left(J_{1}, \mathbb{R}\right) \rightarrow C_{0}\left(J_{1}, \mathbb{R}\right)$ is the truncation operator defined by

$$
(\tau y)(t)=\left\{\begin{array}{l}
\alpha(t), \quad \text { if } y(t)<\alpha(t) \\
y(t), \quad \text { if } \alpha(t) \leq y \leq \beta(t) \\
\beta(t), \quad \text { if } \beta(t)<y(t)
\end{array}\right.
$$

A solution to (3)-(4) is a fixed point of the operator $G$ : $C_{0}\left(J_{1}, \mathbb{R}\right) \rightarrow 2^{C_{0}\left(J_{1}, \mathbb{R}\right)}$ defined by

$$
G(y)=\left\{h \in C\left(J_{1}, \mathbb{R}\right): h(t)=\left\{\begin{array}{cc}
\phi(t), & \text { if } t \in J_{0} \\
\phi(0)+\int_{0}^{t} v(s) d s, & \text { if } t \in J
\end{array}\right\}\right.
$$

where $v \in \widetilde{S}_{F, \tau y}^{1}$ and

$$
\begin{gathered}
\widetilde{S}_{F, \tau y}^{1}=\left\{v \in S_{F, \tau y}^{1}: v(t) \geq v_{1}(t) \text { a.e. on } A_{1} \text { and } v(t) \leq v_{2}(t) \text { a.e. on } A_{2}\right\}, \\
S_{F, \tau y}^{1}=\left\{v \in L^{1}(J, \mathbb{R}): v(t) \in F\left(t,(\tau y)_{t}\right) \text { for a.e. } t \in J\right\}, \\
A_{1}=\{t \in J: y(t)<\alpha(t) \leq \beta(t)\}, A_{2}=\{t \in J: \alpha(t) \leq \beta(t)<y(t)\} .
\end{gathered}
$$

Remark 3.2: $(i)$ For each $y \in C(J, \mathbb{R})$, the set $S_{F, y}^{1}$ is nonempty (see Lasota and Opial [1]).

(ii) For each $y \in C(J, \mathbb{R})$ the set $\widetilde{S}_{F, \tau y}^{1}$ is nonempty. Indeed, by $(i)$ there exists $v \in S_{F, y}^{1}$. Set

where

$$
w=v_{1} \chi_{A_{1}}+v_{2} \chi_{A_{2}}+v \chi_{A_{3}},
$$

$$
A_{3}=\{t \in J: \alpha(t) \leq y(t) \leq \beta(t)\} .
$$

Then by decomposability $w \in \widetilde{S}_{F, \tau y}^{1}$.

We shall show that $G$ is a completely continuous multivalued map, u.s.c. with convex closed values. The proof will be given in several steps.

Step 1: $G(y)$ is convex for each $y \in C_{0}\left(J_{1}, \mathbb{R}\right)$.

Indeed, if $h, \bar{h}$ belong to $G(y)$, then there exist $v \in \widetilde{S}_{F, \tau y}^{1}$ and $\bar{v} \in \widetilde{S}_{F, \tau y}^{1}$ such that

$$
h(t)=\phi(0)+\int_{0}^{t} v(s) d s, \quad t \in J
$$


and

$$
\bar{h}(t)=\phi(0)+\int_{0}^{t} \bar{v}(s) d s, t \in J .
$$

Let $0 \leq k \leq 1$. Then for each $t \in J$ we have

$$
[k h+(1-k) \bar{h}](t)=\phi(0)+\int_{0}^{t}[k v(s)+(1-k) \bar{v}(s)] d s .
$$

Since $\widetilde{S}_{F, \tau y}^{1}$ is convex (because $F$ has convex values) then

$$
k h+(1-k) \bar{h} \in G(y)
$$

Step 2: $G$ sends bounded sets into bounded sets in $c_{0}(J, \mathbb{R})$.

Let $B_{q}:=\left\{y \in C_{0}(J, \mathbb{R}):\|y\|_{\infty} \leq q\right\}$ be a bounded set in $C_{0}(J, \mathbb{R})$ and $y \in B_{q}$, then for each $h \in G(y)$ there exists $v \in S_{F, \tau y}^{1}$ such that

$$
h(t)=\phi(0)+\int_{0}^{t} v(s) d s, t \in J .
$$

Thus, for each $t \in J$ we get

$$
\begin{aligned}
|h(t)| & \leq|\phi(0)|+\int_{0}^{t}|v(s)| d s \\
& \leq\|\phi\|+\left\|\varphi_{q}\right\|_{L^{1}} .
\end{aligned}
$$

Step 3: $G$ sends bounded sets in $C_{0}(J, \mathbb{R})$ into equicontinuous sets.

Let $u_{1}, u_{2} \in J, u_{1}<u_{2}, \quad B_{q}:=\left\{y \in C_{0}(J, \mathbb{R}):\|y\|_{\infty} \leq q\right\}$ be a bounded set in $C_{0}(J, \mathbb{R})$ and $y \in B_{q}$. For each $h \in G(y)$ there exists $v \in \widetilde{S}_{F, \tau y}^{1}$ such that

$$
h(t)=\phi(0)+\int_{0}^{t} v(s) d s, t \in J .
$$

We then have

$$
\begin{gathered}
\left|h\left(u_{2}\right)-h\left(u_{1}\right)\right| \leq \int_{u_{1}}^{u_{2}}|v(s)| d s \\
\leq \int_{u_{1}}^{u_{2}}\left|\varphi_{q}(s)\right| d s .
\end{gathered}
$$

As a consequence of Step 2 and Step 3, together with the Ascoli-Arzela theorem, 
we can conclude that $G$ : $C_{0}(J, \mathbb{R}) \rightarrow 2^{C_{0}(J, \mathbb{R})}$ is a compact multivalued map and, therefore a condensing map.

Step 4: $G$ has a closed graph.

Let $y_{n} \rightarrow y_{0}, h_{n} \in G\left(y_{n}\right)$ and $h_{n} \rightarrow h_{0}$. We shall prove that $h_{0} \in G\left(y_{0}\right) . h_{n} \in G\left(y_{n}\right)$ means that there exists $v_{n} \in \widetilde{S}_{F, \tau y_{n}}$ such that

$$
h_{n}(t)=\phi(0)+\int_{0}^{t} v_{n}(s) d s, t \in J .
$$

We must prove that there exists $v_{0} \in \widetilde{S}_{F, \tau y_{0}}^{1}$ such that

$$
h_{0}(t)=\phi(0)+\int_{0}^{t} v_{0}(s) d s, t \in J
$$

Consider the linear continuous operator $\Gamma: L^{1}(J, \mathbb{R}) \rightarrow C(J, \mathbb{R})$ defined by

$$
(\Gamma v)(t)=\int_{0}^{t} v(s) d s
$$

We have

$$
\|\left(h_{n}-\phi(0)\right)-\left(h_{0}-\phi(0) \|_{\infty} \rightarrow 0 .\right.
$$

From Lemma 2.4, it follows that $\Gamma \circ \widetilde{S}_{F}^{1}$ is a closed graph operator. Also, from the definition of $\Gamma$ we have

$$
h_{n}(t)-\phi(0) \in \Gamma\left(\widetilde{S}_{F, \tau y_{n}}^{1}\right)
$$

Since $y_{n} \rightarrow y_{0}$, it follows from Lemma 2.4 that

$$
h_{0}(t)=\phi(0)+\int_{0}^{t} v_{0}(s) d s, \quad t \in J
$$

for some $v_{0} \in \widetilde{S}_{F, \tau y_{0}}^{1}$.

Step 5: Now, we are going to show that the set

is bounded.

$$
M:=\left\{v \in C_{0}(J, \mathbb{R}): \lambda v \in G(v) \text { for some } \lambda>1\right\}
$$

Let $y \in M$ then $\lambda y \in G(y)$ for some $\lambda>1$. Thus there exists $v \in \widetilde{S}_{F, \tau y}^{1}$ such that

$$
y(t)=\lambda^{-1} \phi(0)+\lambda^{-1} \int_{0}^{t} v(s) d s, t \in J .
$$


Thus

$$
|y(t)| \leq\|\phi\|+\int_{0}^{t}|v(s)| d s, \quad t \in J .
$$

From the definition of $\tau$ there exists $\phi \in L^{1}\left(J, \mathbb{R}^{+}\right)$such that

$$
\left\|F\left(t,(\tau y)_{t}\right)\right\|=\sup \left\{|v|: v \in F\left(t,(\tau y)_{t}\right)\right\} \leq \varphi(t) \text { for each } y \in C(J, \mathbb{R}) .
$$

Thus we obtain

$$
\|y\|_{\infty}=\sup _{t \in J_{1}}|y(t)| \leq\|\phi\|+\|\varphi\|_{L_{1}} .
$$

This shows that $M$ is bounded. Hence, Lemma 2.5 applies and $G$ has a fixed point which is a solution to problem (3)-(4).

Step 6: We shall show that the solution $y$ of (3)-(4) satisfies

$$
\alpha(t) \leq y(t) \leq \beta(t) \text { for all } t \in J_{1} .
$$

Let $y$ be a solution to (3)-(4). We prove that

$$
\alpha(t) \leq y(t) \text { for all } t \in J .
$$

Suppose not. Then there are two cases:

(a) $\quad \alpha(t) \geq y(t)$ for all $t \in J$, and there exists $t^{*} \in J$ such that $\alpha\left(t^{*}\right)>y\left(t^{*}\right)$ and

(b) there exists $t^{*}, t_{*} \in J$ such that $\alpha\left(t^{*}\right)>y\left(t^{*}\right)$ and $\alpha\left(t_{*}\right)<y\left(t_{*}\right)$.

In case $(a)$, from the definition of $\tau$ one has

$$
y^{\prime}(t) \in F\left(t, \alpha_{t}\right) \text { a.e. on } J \text {. }
$$

Thus there exists $v(t) \in F\left(t, \alpha_{t}\right)$ a.e. on $J$ with $v(t) \geq v_{1}(t)$ a.e. on $J$ such that

$$
y^{\prime}(t)=v(t) \text { a.e. on } J .
$$

An integration from 0 to $t^{*}$ yields

$$
y\left(t^{*}\right)-\phi(0)=\int_{0}^{t^{*}} v(s) d s
$$

Since $\alpha$ is a lower solution to (1)-(2), then

$$
\alpha\left(t^{*}\right)-\alpha(0) \leq \int_{0}^{t^{*}} v_{1}(s) d s .
$$

It follows from the facts $\phi(0)=\alpha(0), v(t) \geq v_{1}$ that $\alpha\left(t^{*}\right)<y\left(t^{*}\right)$ which is a contradiction, since $y\left(t^{*}\right)<\alpha\left(t^{*}\right)$.

The case $(b)$ yields also to a contradiction. Consequently,

$$
\alpha(t) \leq y(t) \text { for all } t \in J
$$


Analogously, we can prove that

$$
y(t) \leq \beta(t) \text { for all } t \in J .
$$

This shows that the problem (3)-(4) has a solution in the interval $[\alpha, \beta]$. Since $\tau(y)=y$ for all $y \in[\alpha, \beta]$, then $y$ is a solution to (1)-(2).

\section{References}

[1] Banas, J. and Goebel, K., Measures of Noncompactness in Banach Spaces, Marcel Dekker, New York 1980.

[2] Benchohra, M. and Boucherif, A., On first order multivalued initial and periodic value problems, Dyn. Sys. Appl. 9:4 (2000), 559-568.

[3] Benchohra, M. and Ntouyas, N., On second order differential inclusions with periodic boundary conditions, Acta Math. Univ. Com. LXIX:2 (2000), 173-181.

[4] Deimling, K., Multivalued Differential Equations, De Gruyter, Berlin 1992.

[5] Erbe, L.H., Kong, Q. and Zhang, B.G., Oscillation Theory for Functional Differential Equations, Pure and Applied Mathematics, Marcel Dekker, New York 1994.

[6] Haddock, J.R. and Nkashama, M.N., Periodic boundary value problems and monotone iterative methods for functional differential equations, Nonl. Anal. 22 (1992), 267-276.

[7] Hale, J.K., Theory of Functional Differential Equations, Springer, New York 1977.

[8] Halidias, N. and Papageorgiou, N.S., Second order multivalued boundary value problems, Arch. Math. (Brno) 34 (1998), 267-284.

[9] Henderson, J., Boundary Value Problems for Functional Differential Equations, World Scientific, Singapore 1995.

[10] Hristova, S.G. and Bainov, D.D., Application of the monotone iterative techniques of Lakshmikantham to the solution of the initial value problem for functional differential equations, J. Math. Phys. Sci. 24 (1990), 405-413.

[11] Hu, S. and Papageorgiou, N., Handbook of Multivalued Analysis, Volume I: Theory, Kluwer Academic Publishers, Dordrecht 1997.

[12] Lasota, A. and Opial, Z., An application of the Kakutani-Ky-Fan theorem in the theory of ordinary differential equations, Bull. Acad. Pol. Sci. Ser. Sci. Math. Astron. Phys. 13 (1965), 781-786.

[13] Liz, E. and Nieto, J.J., Periodic boundary value problems for a class of functional differential equations, J. Math. Anal. Appl. 200 (1996), 680-686.

[14] Martelli, M., A Rothe's type theorem for non-compact acyclic-valued maps, Boll. Un. Mat. Ital. 4 (Suppl. Fasc.) (1975), 70-76.

[15] Nieto, J.J., Jiang, Y. and Jurang, Y., Monotone iterative method for functional differential equations, Nonl. Anal. 32:6 (1998), 741-749.

[16] Ntouyas, S.K., Initial and boundary value problems for functional differential equations via the topological transversality method: A survey, Bull. Greek Math. Soc. 40 (1998), 3-41. 


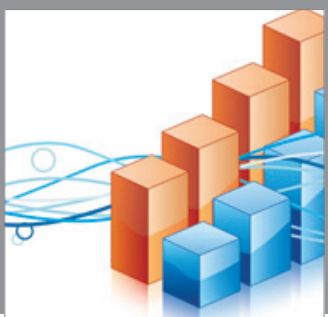

Advances in

Operations Research

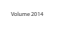

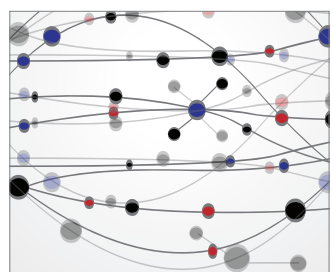

\section{The Scientific} World Journal
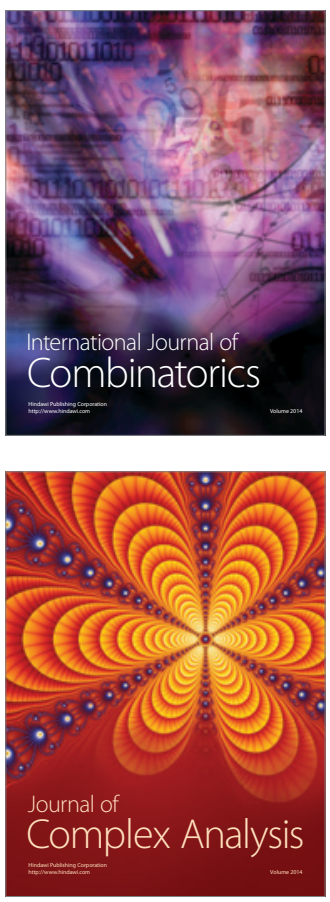

International Journal of

Mathematics and

Mathematical

Sciences
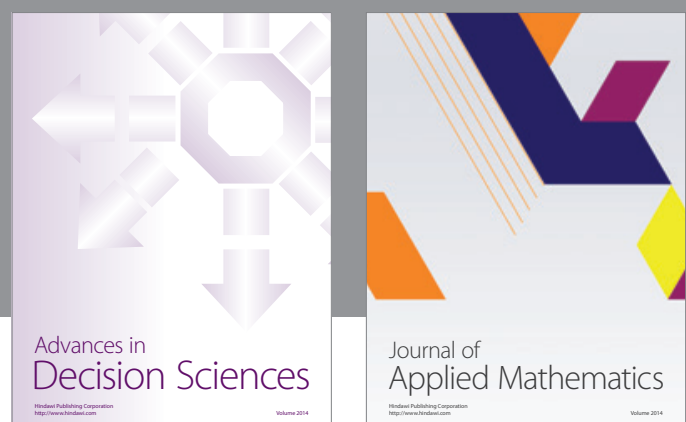

Journal of

Applied Mathematics
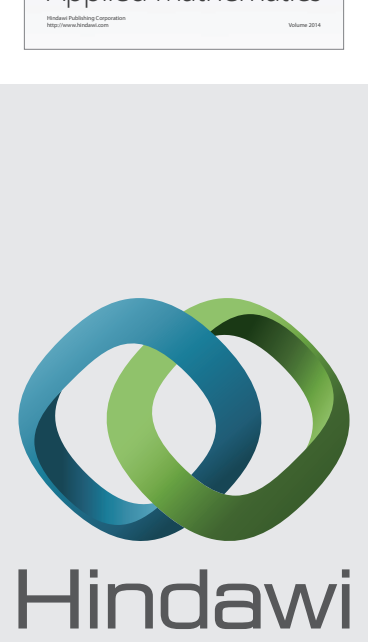

Submit your manuscripts at http://www.hindawi.com
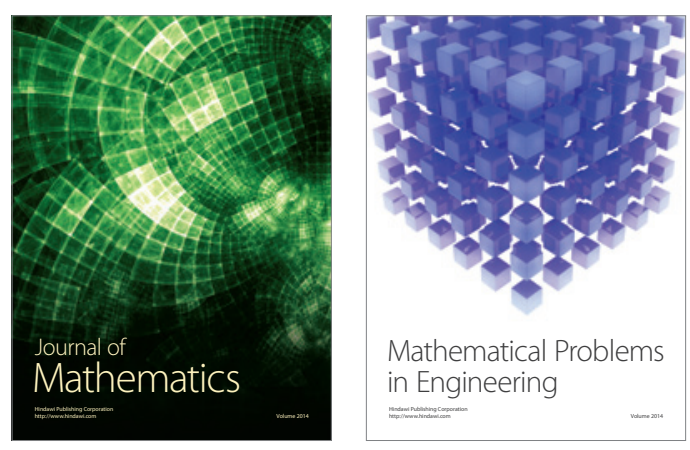

Mathematical Problems in Engineering
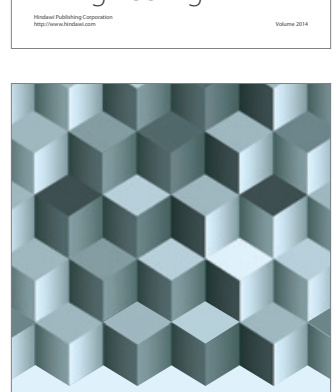

Journal of

Function Spaces
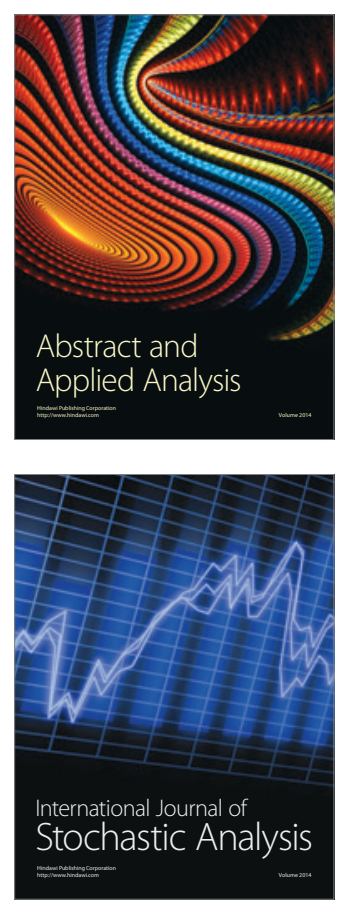

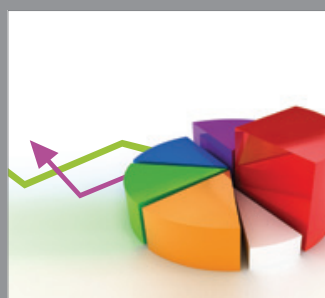

ournal of

Probability and Statistics

Promensencen
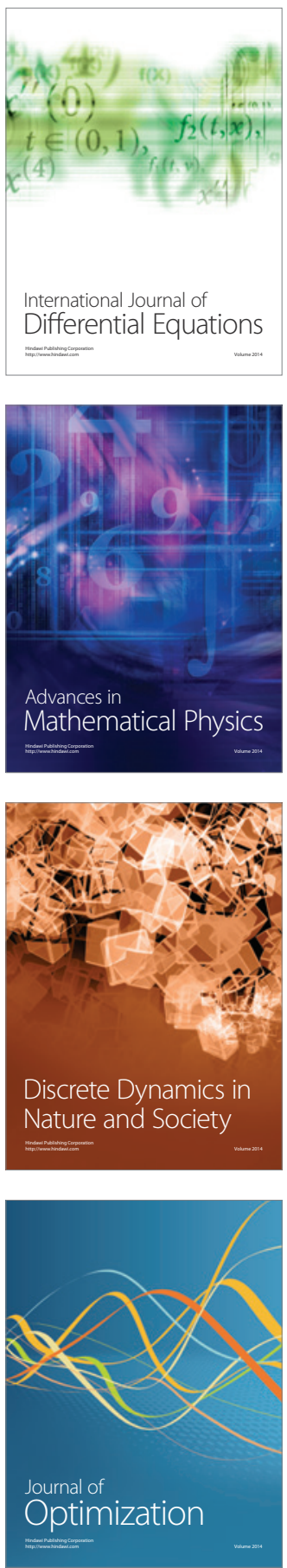